\title{
Evaluation and Management of Neurogenic Thoracic Outlet Syndrome with an Overview of Surgical Approaches: A Comprehensive Review
}

\author{
Christine Lim' \\ Yasaman Kavousi ${ }^{2}$ \\ Ying Wei Lum² \\ Paul J Christo' \\ 'Division of Pain Medicine, Department \\ of Anesthesiology and Critical Care \\ Medicine, The Johns Hopkins University \\ School of Medicine, Baltimore, MD, USA; \\ ${ }^{2}$ Division of Vascular Surgery and \\ Endovascular Therapy, The Johns \\ Hopkins University School of Medicine, \\ Baltimore, MD, USA
}

\begin{abstract}
Neurogenic thoracic outlet syndrome (NTOS) represents a disorder believed to involve compression of one or more neurovascular elements as they exit the thoracic outlet. This comprehensive literature review will focus on the occurrence, classification, etiology, clinical presentation, diagnostic measures, and both nonoperative and operative therapies for NTOS. NTOS represents the most common subtype of thoracic outlet syndrome and can significantly impair quality of life. Botulinum toxin injection into the anterior scalene muscle, or even the middle scalene or pectoralis minor muscles, can reduce the symptoms of this syndrome. The best available evidence for botulinum toxin therapy to the cervicothoracic muscles supports the value of this treatment for reducing pain in the affected extremity, and for an approximate duration of 2 months or more. Surgical approaches and newer minimally invasive surgical approaches offer high rates of improvement in select centers.

Keywords: thoracic outlet syndrome, neurogenic thoracic outlet syndrome, anterior scalene muscle, brachial plexus, compression neuropathy
\end{abstract}

\section{Introduction}

The term "thoracic outlet syndrome" (TOS) was coined in 1956 by Peet et al ${ }^{1}$ and is used to describe compression of one or several neurovascular structures (ie brachial plexus, subclavian artery or vein or axillary artery or vein) that cross the thoracic outlet. This group of disorders with a presumed compressive or nerve entrapment etiology has been described by multiple terms that reflect either the region of pathology or the maneuver that precipitated the symptoms. ${ }^{2}$ Some of these prior terms include "cervical rib and band syndrome", "scalenus anticus syndrome," and "pectoralis minor syndrome". 2 The most vulnerable structures to compression are generally understood to be the brachial plexus and the subclavian vessels. Three areas of compression likely exist: scalene triangle, costoclavicular space, or the pectoralis minor space. ${ }^{3,4}$ TOS can be further subclassified based on whether the etiology involves the vasculature (e.g. venous or arterial) or the brachial plexus (e.g. neurogenic).

Neurogenic thoracic outlet syndrome (NTOS) can present with a wide variety of symptoms including ipsilateral upper extremity pain, sensory loss, paresis, edema, shoulder and neck discomfort, and even sympathetic nervous impairment. NTOS symptoms can range from mild to severe; however, if left untreated, NTOS is associated with significant detrimental impacts on functional status and quality of
Correspondence: Paul J Christo

Email pchristo@jhmi.edu 
life. For example, the impact matches those suffering from chronic heart failure. ${ }^{5}$ Unfortunately, because there is a wide spectrum of presenting symptoms, there are no uniform criteria for diagnosis, treatment, patient selection, or treatment approaches. ${ }^{6}$ Further, no definitive test exists for TOS, which may suggest that TOS is relatively underdiagnosed. This lack of consensus and poor physician awareness (and therefore recognition) make it difficult to estimate the true incidence of TOS, ${ }^{7}$ though several articles report an incidence of $3-80 / 1,000,{ }^{8}$ while Roos describes an incidence of 0.3 and $2 \%{ }^{7,9}$ Interestingly, even these incidences are disputed, with a more recent study analysis suggesting that NTOS may be considered a "rare" disorder, with an incidence of 2-3/ 100,000 per year. $^{7}$

In addition to the controversy surrounding the diagnosis of NTOS, the treatment of NTOS also lacks consensus. This comprehensive literature review focuses on the classification, etiology, clinical presentation, diagnostic measures, and treatment of NTOS, including nonoperative therapies such as physical modalities, pharmacological therapies, minimally invasive therapies including intramuscular botulinum toxin, and surgical interventions.

\section{Search Strategy}

This article intends to provide readers with the most relevant and up-to-date information regarding the diagnosis and treatment of NTOS. In the first phase of the search, a comprehensive electronic literature search (1956-2021) process was conducted through PubMed using the MeSH term: "thoracic outlet syndrome" with the following filters: article types (meta-analysis; systematic reviews), text availability (abstract available), publication dates (from January 1 2006 to January 30, 2021), species (humans), and language (English). Following this methodology, we found 0 metaanalysis and 6 systematic reviews. Due to the scarce literature of meta-analysis on the topic, other articles were included, including a literature search process that included PubMed, Clinical Key, and Google Scholar. References cited in relevant articles were also reviewed. Search terms and criteria were further globalized to include clinical trials, reviews, multicenter studies, guidelines, and practice guidelines. For each article, pertinence was considered. Articles solely focused on vascular TOS were excluded.

\section{Classification}

There are three generally recognized forms of TOS that are classified based on their pathophysiology: neurogenic (secondary to presumed brachial plexus compression), arterial (subclavian artery compression), and venous (subclavian vein compression). ${ }^{10}$ Neurogenic TOS accounts for the vast majority of TOS cases, with studies generally estimating about $90 \%$, but as high as $95 \% .{ }^{10}$ Neurogenic TOS can be further subclassified as "true" NTOS, also known as "classic TOS" or "cervical rib and band syndrome"; 2 and "nonspecific," which is also known as "common," or "disputed" NTOS. ${ }^{6,11,12}$ Classic NTOS is associated with objective findings including a complete or incomplete cervical rib with a tight band and accompanied electrodiagnostic (EDX) changes. Classic NTOS represents a minority of patients and affects just $1 \%$ of all NTOS cases. ${ }^{9,13}$ The common or nonspecific type is defined as having symptoms suggestive of brachial plexus compromise, but without objective findings, and accounts for $95-99 \%$ of all neurogenic cases. ${ }^{12}$ When discussing the neurogenic form of TOS, we are referring to the nonspecific type unless otherwise stated.

These groups (NTOS, VTOS, and ATOS) can be further subclassified based on their etiologies, including congenital, functionally acquired, or traumatic causes. For example, congenital causes include an elongated $\mathrm{C} 7$ transverse process, cervical first rib, or other structural abnormalities such as fibrous bands. ${ }^{2,9}$ Functionally acquired causes can be due to repetitive motion, such as frequent overhead movements, typing, and assembly line work, or athletes participating in volleyball, swimming, or baseball for instance. Traumatic causes include whiplash injuries (hyperextension-flexion injury), motor vehicle accidents, and falls. ${ }^{12}$

\section{Anatomy, Etiology, and Pathophysiology}

The thoracic outlet encompasses the space from the supraclavicular fossa to the axilla. The brachial plexus and the subclavian vessels are vulnerable to compression as they cross three areas: (1) the interscalene triangle, (2) the costoclavicular triangle, (3) the subcoracoid space. ${ }^{9}$ Neurovascular compression more commonly occurs in the proximal regions such as the interscalene triangle and the costoclavicular triangle. ${ }^{14}$

The interscalene triangle is bordered by the anterior scalene muscle anteriorly, the middle scalene posteriorly, and the medial surface of the first rib inferiorly. ${ }^{13}$ The vast majority of cases of TOS involve neural and/or vascular compression within the interscalene triangle. ${ }^{15}$ Notably, 
the trunks of the brachial plexus and subclavian artery lie within the triangle; however, the subclavian vein travels anteriorly and posteriorly to the anterior scalene muscle and joins the internal jugular vein to form the brachiocephalic vein. ${ }^{16}$

The costoclavicular space is bordered by the clavicle superiorly, inferiorly by the first thoracic rib, and posteriorly by the anterior scalene muscle at its insertion site. ${ }^{2}$ Its components include the brachial plexus at the level of the cords, as well as the subclavian artery and vein. ${ }^{12}$

The subcoracoid or retropectoralis minor space $e^{4,12,17}$ is bordered by the pectoralis minor muscle anteriorly, posteriorly by the 2nd-4th ribs, and superiorly by the coracoid process. Its contents include the axillary artery and vein, and the brachial plexus generally at the level of the branches. $^{12}$

There may be varying etiologies involved in NTOS. Brantigan and Roos ${ }^{9}$ suggest that there may be congenital abnormalities with superimposed trauma, muscle spasm, and fibrosis leading to a "space problem." This "space problem" caused by congenital anomalies such as cervical ribs and fibrotic bands can result in compression of the brachial plexus and ultimately symptomatology. ${ }^{6}$ Notably, the structure most implicated in the symptomatology of NTOS is the anterior scalene muscle. ${ }^{2,6,9}$ Interestingly, histologic studies demonstrate that injury to either the anterior scalene muscle or the middle scalene muscle may be the most likely causative factor. ${ }^{18,19}$ Moreover, examination of these excised scalene muscles reveals significant muscle fibrosis and the presence of three times more scar tissue compared to controls.

Nerve conduction anomalies occur in patients with the classic form of NTOS that leads to muscle atrophy, although a definitive pattern of neurophysiological changes has not been identified. ${ }^{20}$ In contrast, consistent, reproducible findings of pathological dysfunction in the brachial plexus or scalene muscles have yet to be uncovered for nonspecific NTOS. Neuroimaging and electrodiagnostic studies fail to demonstrate consistent neurophysiological dysfunction; ${ }^{20}$ however, there is some evidence of a compressive etiology at least in some patients. For instance, 3T MR neurography in seven out of 30 patients with a diagnosis of NTOS showed the presence of an anatomical structure compressing the brachial plexus which surgical exploration confirmed. ${ }^{21}$

The anterior scalene muscle (ASM) originates from the anterior tubercles of the transverse processes of the C3-C6 vertebrae and inserts into the scalene tubercle of the first rib. In a study by Swank and Simeone ${ }^{22}$ that evaluated "scalenus anticus" syndrome in 1944, all patients demonstrated hypertrophy of the anterior scalene muscle. The ASM acts as an accessory muscle of respiration that raises the first rib and assists in bending and rotating the neck. ${ }^{13}$ Congenital anomalies located where the ASM inserts along the first rib, or an abnormally broad insertion of the ASM to the scalene tubercle can predispose a patient to the development of NTOS. Brantigan and $\operatorname{Roos}^{9}$ note that in addition to the anatomical variants that predispose patients to developing NTOS, there may also be superimposed injury that precipitates onset of symptoms. Injuries such as trauma or whiplash, blows to the shoulders, or repetitive trauma can all result in muscle spasm of the ASM. In turn, these injuries may cause edema, and exacerbate the "space problem" in NTOS. Further, scar development and fibrotic changes may contribute to neural compromise of the brachial plexus, thus perpetuating the pain cycle. It is for this reason that many surgical and minimally invasive treatments target the ASM. Specifically, these interventions aim to relieve presumed neural compromise of the brachial plexus by easing tension or spasm of the muscle. ${ }^{6}$

\section{Clinical Presentation}

The clinical presentation of TOS varies depending on the type, ie NTOS vs VTOS vs ATOS. Especially within the NTOS population, the clinical presentation can be diverse. The presentation can range from mild discomfort that is exacerbated by certain upper extremity or neck positions to the more common description of pain, paresthesias and/ or numbness in the fingers, hands, forearm, or arm on the affected side.

In general, NTOS occurs more frequently in women (3-4:1), and typically presents between the ages of 20-40 years. ${ }^{6,9,23}$ Furthermore, certain vocations such as violinists, jobs that involve significant computer usage in nonergonomic positions, and assembly line workers appear vulnerable given the physical nature of their occupation. Another population at risk includes athletes incorporating repetitive overhead motions such as volleyball players, swimmers, pitchers, and weightlifters.

Symptoms and clinical features include a history of neck trauma, whiplash injury, or repetitive overhead motions, pain exacerbated with certain positions, and pain often consistent with nerve irritation including complaints of pain, paresthesias, and weakness in the arm and hand. ${ }^{6,10,23}$ In general, these symptoms can involve all five 
fingers, and can even occur in the entire upper extremity in a non-dermatomal pattern. The symptoms can be further subdivided based on the areas of possible dysfunction. This includes compression of the upper brachial plexus, which involves compression of the $\mathrm{C} 5-\mathrm{C} 7$ nerve roots, causing complaints of ipsilateral neck, trapezius, mastoid or anterior chest wall pain, or even occipital headaches. ${ }^{6}$ In contrast, compromise of the lower plexus, involving the C8-T1 nerve roots can result in symptoms around the posterior aspect of the shoulder, with radiculopathy down the arm in the ulnar distribution. ${ }^{12}$ Despite these specific subdivisions, patients often feel that the entire limb is affected without dermatomal preference, which contributes to the controversy and difficulty surrounding the diagnosis.

\section{Diagnosis}

\section{Diagnosis - History}

Although NTOS is the most common subtype, representing up to $95 \%$ of TOS diagnoses, it is also the most controversial because it is difficult to diagnose, and remains without objective measures/criteria for diagnosis. ${ }^{6,24}$ As a result, the diagnosis remains one of exclusion, and relies heavily on a detailed and thorough history and physical examination. ${ }^{6,10}$ Unlike ATOS and VTOS, which can be diagnosed using ultrasound or CTA, objective measures and testing procedures for diagnosing NTOS remain limited. Further obscuring the diagnosis is confounding diagnoses that present with similar symptoms. For instance, patients who present with neck and arm pain may be misdiagnosed with cervical radiculopathy, rotator cuff injury, or peripheral nerve entrapment.

More recently, the Society for Vascular Surgery developed reporting standards for Thoracic outlet syndrome, including standardized nomenclature, definitions, and more consistent reporting standards. The most recent definition from 2016 by Illig et ${ }^{4}$ defines NTOS as the presence of three of the four criteria:

1. Local symptoms in an area of compression (e.g., chest wall, trapezius, neck) and confirmed with pain on palpation in the specific area

2. Peripheral findings of arm or hand symptoms based on history, and confirmed by provocative maneuvers that reproduce or worsen the symptoms.

3. Absence of other reasonable/likely diagnoses such as cervical, shoulder, or peripheral nerve pathology.

4. Positive response to a scalene injection (typically local anesthetic)
Patients may also note a history of trauma. ${ }^{14}$ It is important to evaluate occupational, avocational, or sports activity risk factors. ${ }^{4,6,12}$ Other key symptoms that can be obtained from a thorough history include complaints of pain, numbness, tingling, sleep disturbances, and weakness, while exacerbating factors can include exercising, overhead arm motion, and driving. ${ }^{4,6,12}$ Symptom distribution generally involves all five fingers but can be more pronounced in the fourth and fifth digits and the ulnar forearm. ${ }^{11}$ Symptoms can sometimes be distinguished from cervical disc syndrome since NTOS generally presents without dermatomal preference, while cervical stenosis or disc syndrome may often present with a more defined dermatomal pattern; however, this can also make it difficult for general physicians, as the patient may complain of a nonspecific clinical presentation thereby confounding the picture and leading to an inaccurate or delayed diagnosis. ${ }^{6}$

\section{Diagnosis - Physical Exam}

In addition to obtaining a history, a complete examination helps rule out other diagnoses. This includes observation of posture, bulk, and tone of muscular components including the ipsilateral shoulder, arm, and hand as well as a vascular, neurologic, and motor exam. What is typically regarded as "true-type" NTOS can lead to muscular atrophy including the "Gilliatt-Sumner hand", described as wasting of the thenar eminence, interossei muscles, and the abductor pollicis brevis. ${ }^{15}$ However, these objective findings are not found in the nonspecific form of NTOS. Finally, provocative and stress maneuvers can be performed. In NTOS, thumb pressure applied over the brachial plexus in the region of the supraclavicular fossa or scalene muscles for a few seconds may reproduce patient's symptoms, leading to pain in the neck, shoulder, or radicular symptoms down the ipsilateral arm. ${ }^{6}$ Two provocative tests that may better aid in the diagnosis of NTOS, both of which offer relatively high sensitivity, include the elevated arm stress test (EAST), and the upper limb tension test (ULTT). ${ }^{4,12}$

The EAST test, also known as the Roos test, is designed to reproduce symptoms that occur when the scalene triangle narrows. ${ }^{25}$ The patient is seated with shoulders abducted 90 degrees and in external rotation, with elbows flexed to 90 degrees. Interestingly, the length of time to perform the test is debated - some publications suggest maintaining the position for one minute, ${ }^{25}$ while 
others including the article by Brantigan and Roos ${ }^{9}$ suggest maintaining the position for 3 minutes. A positive test result precipitates the onset of pain, paresthesias, heaviness, or weakness of the ipsilateral extremity.

The ULTT is also known as the Elvey test, ${ }^{12}$ and is performed by stretching the brachial plexus. The position involves a stepwise movement, starting with arms abducted and elbows at 90 degrees. If the patient remains asymptomatic, the arm is extended at the elbow, and hands are pronated. Finally, the wrists are extended, and the head is tilted away from the arm being tested. ${ }^{12,25-27}$ The test is considered positive if at any point during the stepwise positioning, the patient reports the onset or exacerbation of neck and/or arm pain.

In addition to the EAST and ULTT test, the Adson's maneuver is commonly performed to distinguish or elicit a response in the setting of a vascular-type thoracic outlet syndrome. This maneuver is accomplished by extending the neck and rotating the head towards the symptomatic side while holding deep inspiration. The test is considered positive if there is a reproduction of symptoms or loss of radial pulse. ${ }^{11,12,17,28}$

These neurogenic and vascular provocative maneuvers are often used together to improve specificity: the Adson's and EAST test when used alone have a specificity of $76 \%$ and $30 \%$, respectively, while performing the two together yields a specificity of $82 \%$ for general TOS (not subclassified). ${ }^{29}$

\section{Diagnosis -Diagnostic Testing Imaging}

Although imaging, particularly ultrasonography, can provide support for diagnosing vascular forms of TOS, the efficacy of imaging modalities in NTOS remains unclear. However, imaging studies such as CT, chest radiography, MRI, and ultrasound are frequently used to detect underlying conditions that could cause the observed symptomatology. For example, CT and chest radiography can identify an elongated $\mathrm{C} 7$ transverse process or the presence of a cervical rib. ${ }^{25,26}$ These studies can also be informative for possible surgery regarding TOS subtypes (eg vascular vs neurogenic), as well as patient's anatomy of the thoracic inlet including scalene musculature, ligamentous bands, and first rib. ${ }^{25}$

MRI has also been considered as a potentially useful diagnostic measure for NTOS; however, its utility is dependent on the technique utilized. ${ }^{30}$ MRI specificity and sensitivity are $41 \%$ and $33 \%$ respectively based on one study of 42 cases of TOS, but not NTOS however. ${ }^{31}$ In contrast, MR Neurography (MRN) may be a more beneficial tool for imaging any abnormalities associated with NTOS. ${ }^{21}$ In particular, high-resolution scanners with 3.0 $\mathrm{T}$ magnetic field strength, with a focus on Short Tau Inversion Recovery (STIR) sequence and Spectral Adiabatic Inversion Recovery (SPAIR) module can provide a more complete anatomical description of the nerves of the brachial plexus. ${ }^{30}$

\section{Electrodiagnostic Testing}

Historically, electrodiagnostic studies were used to stratify or subclassify NTOS into "true" vs "nonspecific" NTOS. However, since the advent of the Journal of Vascular Surgery reporting standards in 2016, this distinction has been phased out. ${ }^{4}$ Hence, the role of electrodiagnostic testing including nerve conduction studies and needle electromyography in the diagnosis of NTOS remains unclear. However, a study by Tsao et al in 2014 found that testing the medial antebrachial cutaneous nerve and median motor nerve supplying the abductor pollicis brevis commonly revealed conduction deficits in both nerves, which derive from C8 and T1 fibers. Findings were abnormal in $89 \%$ of patients with NTOS when electrodiagnostics were combined with medial antebrachial cutaneous nerve and median motor nerve testing. ${ }^{32}$ This may suggest a role for electrodiagnostic testing in distinguishing NTOS from other neuropathies, and specifically identifying peripheral neuropathies as a possible etiology of the patient's symptoms. ${ }^{30}$

\section{Local Anesthetic Injection}

Another diagnostic measure that is perhaps even more valuable, given the most recent 2016 guidelines by the Journal of Vascular Surgery is an injection of local anesthetic, typically into the scalene muscles. This technique was first described in $1939,,^{33}$ and has been incorporated as a supportive diagnostic maneuver for NTOS. The injection can assist with diagnosis and help predict positive surgical outcomes for NTOS. ${ }^{6,30,34}$ It is hypothesized that the intramuscular anterior scalene block acts by temporarily blocking or paralyzing the muscle in spasm, thereby allowing the first rib to descend to decompress the thoracic outlet. $^{6,35,36}$ One study by Weaver et al in 2019 showed that after injection of local anesthetic, there was a small but statistically significant change in baseline height of the anterior scalene muscle in patients who responded to the block. ${ }^{24}$ The anterior scalene muscle injection can be performed with anatomical landmarks, ${ }^{33,37} \mathrm{EMG},{ }^{38}$ 
ultrasonography, ${ }^{35}$ EMG and fluoroscopy, ${ }^{34}$ EMG and ultrasonography, ${ }^{34} \mathrm{CT}^{6}{ }^{6}$ and $\mathrm{MRI}{ }^{24} \mathrm{~A}$ retrospective review suggests that $\mathrm{CT}$ guidance can minimize the rate of complications such as Horner's syndrome, dysphonia, brachial plexus block, and dysphagia. ${ }^{6,36,39}$ Diagnostically, injection of local anesthetic into the ASM could be used as a confirmatory maneuver if the patient reports relief. ${ }^{4,6,30,40}$

\section{Treatment}

There is a wide range of treatment options including lifestyle changes and conservative approaches as well as surgical intervention. The consensus for appropriate progression from conservative to interventional treatment options remains controversial, although most physicians suggest physical therapy as an initial first step.

\section{Conservative Treatment}

In general, conservative management focuses on a multimodal approach with a combination of patient education, physical therapy, behavior modification, and cognitive behavioral therapy. ${ }^{6,12}$

\section{Physical Therapy}

Traditionally, physical therapy focuses on exercises that aim to create and increase the space between the first rib and clavicle, and decrease the tensile load of the limb. Additionally, it targets postural retraining and optimizing diaphragmatic breathing to reduce accessory muscle overuse that can contribute to compression of the thoracic outlet. ${ }^{41}$ One study by Balderman et al in 2019 showed that $27 \%$ of patients with NTOS obtained satisfactory improvement with physical therapy alone, ${ }^{42}$ while another study demonstrated symptomatic relief in 25 of 42 patients after 6 months of physical therapy. ${ }^{17}$

More recently, physical therapists are applying novel techniques in neuroscience and cognitive behavioral in the form of informed physical therapy for improving outcomes. ${ }^{41}$ For example, physical therapists strive to improve patient function and symptoms through several techniques that target various domains: external support such as a shoulder girdle, usually used in the initial short term to help control symptoms, function and ergonomics (such as postural training), neural glides, and psychologically informed physical therapy. $^{41}$

\section{Medications}

Pharmacological strategies can help ease painful symptoms. Those that treat an underlying spasm symptomatology include muscle relaxants (e.g. tizanidine), NSAIDS (e. g. meloxicam or ibuprofen), and trigger point injections containing local anesthetic to the trapezius muscle most frequently. ${ }^{6,16}$ Given the presentation of neuropathic symptoms in NTOS (eg, radicular pain, sensory deficits, burning pain), serotonin-norepinephrine reuptake inhibitors (e.g. duloxetine), tricyclic antidepressants (eg nortriptyline), and $\alpha 2 \delta$ calcium channel ligands (e.g. gabapentin) can be considered as adjuncts or even primary therapies for pain control. Opioids including short acting agents, or sustained-release formulations of morphine or oxycodone, transdermal fentanyl, or methadone can be trialed as well; however, opioid therapy should be reserved for pain that impairs quality of life significantly and that persists despite appropriate trials of other pharmacotherapies, physical therapy, minimally invasive therapies, and/or surgery. ${ }^{6}$

\section{Minimally Invasive Treatment}

There is evidence that supports the administration of botulinum toxin (BTX) to the cervicothoracic musculature as a treatment for NTOS. ${ }^{36,40,43,44}$ This toxin is produced by Clostridium botulinum, which can cause severe muscle weakness, nervous system dysfunction, and respiratory distress if poisoning occurs. ${ }^{6,36}$ BTX has 7 known serotypes, two of which are approved by the FDA for clinical use in humans: BTX type A and $\mathrm{B}^{43}$ All types cleave SNARE proteins, thereby preventing the synaptic fusion that promotes release of neurotransmitters for muscle contraction (eg acetylcholine). ${ }^{43}$ Ultimately, the therapeutic use of this toxin results in reduced muscle overactivity, and possibly decreased pain and inflammation. ${ }^{6,45}$ Other approved uses of botulinum toxin include hemifacial spasm, blepharospasm, strabismus, cervical dystonia, and chronic migraine. ${ }^{46}$ At present, the use of botulinum toxin has expanded to include treatment of cervicothoracic muscular pain, lumbosacral myofascial pain, piriformis syndrome, lateral epicondylitis, and neurogenic thoracic outlet syndrome. ${ }^{43}$

In NTOS, injection of BTX-A (eg onabotulinum toxin A) into the ASM, MSM, or even the pectoralis minor muscle can be a beneficial therapeutic intervention. One study suggests that BTX-A can also be used to predict patients who will respond positively to operative intervention. For instance, a recent study demonstrated that a better 
response to BTX injections correlated positively with a better response to surgery (generally first rib resection and scalenectomy) with a high degree of specificity (90\%). This same study also noted that there was a positive predictive value of $99 \%$ for patients who responded to BTX and subsequently responded to surgery. ${ }^{40}$ Therapeutically, a retrospective analysis of 41 patients with suspected NTOS who underwent 92 BTX injections in total (58 ASM, 33 pectoralis minor, and 1 subclavius) showed a $69 \%$ rate of pain relief. ${ }^{44}$ The mean time to symptom improvement was 12 days, with an average duration of 31 days (range of 5-75 days). ${ }^{44} \mathrm{~A}$ prospective analysis by Christo et $\mathrm{al}^{36}$ showed significant pain reduction at two months with extension to three months.

The technique of BTX-A injection into the ASM or pectoralis minor has been described with various approaches, similar to those used for diagnostic local anesthetic blockade. Several studies describe a CT-guided technique for ASM injection, which affords an efficient, safe, and reliable method of needle insertion and local anesthetic or BTX-A delivery. ${ }^{36}$ The additional benefit of $\mathrm{CT}$ in comparison to EMG or fluoroscopic guidance includes the ability to visualize local structures that are important to avoid while performing the procedure. Moreover, this allows for real-time imaging capabilities when performing the injection. In a retrospective analysis of 106 patients, or 146 scalene injections, including 63 BTX-A injections, CT guidance minimized undesired side effects including Horner syndrome, dysphonia, BP block, and dysphagia. ${ }^{39}$

To date, there is only one double-blind, randomized, controlled trial of NTOS with BTX-A by Finlayson et al in $2011 .^{47}$ This study evaluated a total of 38 patients (20 in the treatment arm, 18 in the placebo arm) and compared the primary outcome measure of change in VAS scores for pain at 6 weeks. The BTX-A group received 37.5 units in the anterior scalene and 37.5 units in the middle scalene for a total of 75 units, while the placebo group received a saline injection. Although the VAS scores between placebo and BTX-A failed to find a significant difference between them, the VAS scores did show a change from baseline of $5.03 \mathrm{~mm}$ in favor of the BTX-A group at 6 weeks. The needles were placed using EMG guidance in contrast to other practitioners who perform this procedure under ultrasound or CT guidance. Furthermore, the study's population had a mean duration of symptoms that varied largely between the BTX-A and the placebo control arm with a mean pain duration of 6 years and 3 years, respectively. It is speculated that this increased duration and delay in treatment prior to their diagnosis may have contributed to the development of chronic pain with associated central sensitization. Also, in both groups, there was possibly a floor effect due to a low baseline pain score (5mm in the BTX-A group and $14 \mathrm{~mm}$ in the placebo group). Because this study was underpowered, a true distinction between placebo and the treatment group of VAS scores could not be determined. Finally, this study may have been subject to allocation bias from suboptimal blinding methods.

\section{Surgical Interventions}

Surgical intervention for thoracic outlet syndrome (TOS) depends largely on the specific type of thoracic outlet syndrome. For instance, in cases of neurogenic TOS (nTOS), surgical intervention is offered to those who have failed conservative management. On the other hand, patients with arterial TOS (aTOS) most often require surgery as the initial step in their treatment. Patient selection is particularly important in the success of the operative management of TOS. In a retrospective study done at our institution, 538 patients who underwent first rib resection (FRR) for all three forms of TOS were studied in a tenyear period. In the span of a decade, when comparing the first and second halves of the study period, improved or fully resolved symptoms increased from $93 \%$ to $96 \%$. More vTOS patients and fewer nTOS patients were treated during this time. These findings were likely due to changes in surgical practice, appropriate selection of patients with nTOS, use of a standard protocol for patients with vTOS, and expedient intervention in patients with aTOS. ${ }^{48}$

Patients with neurogenic TOS who are refractory to conservative management should be considered for decompression of brachial plexus by means of resection of the cervical rib, the first rib or other anomalies that may be causing impingement in the thoracic outlet. This is often combined with scalenectomy or scalenotomy and brachial plexus neurolysis. Decompression of the brachial plexus is accomplished via different approaches including transaxillary, supraclavicular, and infraclavicular techniques. All three techniques have comparable results in high-volume centers. The ideal approach should be based on patient's anatomy and symptoms, as well as the surgeon's operative experience.

In our practice, surgical decompression of the thoracic outlet is achieved via a transaxillary approach. The transaxillary approach was first described by Roos in $1966 .{ }^{49}$ 
Our institutional studies suggest greater than $90 \%$ rate of improvement in symptoms using this technique. ${ }^{50}$ The transaxillary approach is advantageous, as it allows for excellent visualization and exposure of the anterior portion of the first rib, where the compression occurs. In this approach, the patient is placed in the lateral decubitus position, and the arm of the affected side is placed in a Machleder retractor. A transverse skin incision is made at the lower border of the axillary hairline, which extends from the anterior border of the latissimus dorsi to the lateral edge of the pectoralis major. The chest wall is encountered above the serratus fascia. Subclavius muscle, subclavian vein, anterior scalene muscle, subclavian artery, brachial plexus and middle scalene muscles are then identified. The first rib is then dissected circumferentially using a large periosteal elevator and freed from the intercostal muscle fibers and the middle scalene muscle fibers. If a cervical rib is present, the periosteal elevator is used again to remove any muscle fibers from the rib. Care is taken not to enter the pleural space. A bone cutter is then used to divide the rib anteriorly and posteriorly. The rib should be cut behind the nerve root without causing injury to the nerve root. This usually requires the rib to be cut more than once. Blunt and sharp dissection are then used to dissect between the subclavian artery and the trunks of the brachial plexus to identify the roots of the brachial plexus, and to remove any fibroconnective tissue in between the trunks.

Venous TOS causes compression and thrombosis of the axillosubclavian vein. This is also known as effort thrombosis or Paget-Schroetter syndrome. In cases of vTOS, the mainstay of treatment consists of one or a combination of the following: anticoagulation, thrombolysis, and surgical decompression. Despite the complex nature of the disease, if it is addressed expeditiously, it has excellent outcomes. One of the main predictors of outcomes is the initiation of treatment within 14 days of symptoms onset. ${ }^{51}$ Historically, the treatment has been centered around anticoagulation with relatively poor outcomes. In recent years, catheter-directed thrombolysis has become the initial management strategy in the treatment of vTOS, and has high rates of success in re-establishing luminal patency if performed within 14 days of symptoms. ${ }^{52}$ This is accomplished by percutaneously accessing the vein, and inserting a catheter in an antegrade fashion into the thrombus. If the lesion can be crossed, a catheter with side ports is placed across the area of thrombosis, and tissue plasminogen activator ( $r-\mathrm{tPA}$ ) is infused via the catheter for up to
48 hours. If the lesion cannot be crossed, attempts at mechanical aspiration of the thrombus are made with a catheter with an endport. This tends to be less successful in re-establishing patency. ${ }^{51}$ Repeat venography should be performed after these procedures to assess luminal patency. Although the short-term outcomes of catheterdirected thrombolysis including reduced pain, swelling, and luminal patency are excellent, it is not the definitive treatment for vTOS, with up to $23 \%$ rate of re-thrombosis in 13 months following thrombolysis. ${ }^{53}$ FRR with scalenectomy is therefore advocated to achieve durable results. In our institution, after surgical decompression, patients are discharged home on therapeutic anticoagulation. They will then return after 2-3 weeks for a follow-up venography. Anticoagulation is discontinued if the vein is found to be patent. Angioplasty is used in cases where the vein is patent but has intrinsic defects.

Arterial TOS can manifest either as symptomatic in cases of ischemia and embolization, or asymptomatic in cases of aneurysmal degeneration, occlusion or silent embolization. Surgical treatment is indicated for all symptomatic patients. The main tenants of treatment in cases of arterial TOS include removing the source of arterial compression and arterial embolus and restore distal perfusion. Therefore, the first principle of treatment involves decompression of the thoracic outlet by means of FRR and scalenectomy. Patients who have upper extremity ischemia with any degree of motor deficit or significant sensory deficit due to distal embolization should undergo immediate operation including thrombectomy. In patients with chronic embolization, bypass surgery may be necessary. Thrombolysis may be appropriate in cases of mild ischemia. Subclavian artery aneurysms associated with aTOS have traditionally been treated with open repair. However, reports of endovascular repair of subclavian artery aneurysm have shown promising results. ${ }^{54}$

The approach to first rib resection varies among surgeons and institutions, with no single approach regarded as the standard of care. Although open surgical approach is the most prevalent technique, there has been several reports describing thoracoscopic and video-assisted techniques with successful outcomes for neurogenic, venous, and arterial TOS. Minimally invasive techniques including video-assisted thoracoscopic first rib resection (VATS), robotic-assisted transthoracic first rib resection, and endoscopic transaxillary first rib resection have been described in the literature. Soukiasian et $\mathrm{al}^{55}$ reported a retrospective case series of 66 VATS procedures for FRR performed on 58 patients, $80 \%$ of which were 
performed for nTOS, with complete resolution of symptoms in $89 \%$ of the patients. In contrast to the traditional transaxillary technique, VATS allows for less traction on the arm and better visualization of the neurovascular bundle and the attachments to the scalene muscle. ${ }^{55}$

Gharagozloo et $\mathrm{al}^{56}$ described the results of 83 robotic FRR performed for vTOS from a single surgeon. Robotic FRR resulted in subclavian vein patency in $69 \%$ of the patients, while $25 \%$ of the patients had residual stenosis requiring balloon angioplasty. The remainder of patients required angioplasty and stenting of the subclavian vein. In this cohort, patients experienced greater pain and required drainage of the chest, which resulted in a longer hospitalization. The outcome of this study was comparable to FRR with VATS. ${ }^{56}$ Endoscopic transaxillary FRR has also been described as a safe and effective technique in the management of TOS. Abdellaoui et $\mathrm{al}^{57}$ performed 28 endoscopic-assisted transaxillary FRR, $85 \%$ of which was performed for nTOS. This was done through a single 6-7 cm incision, without lung collapse. In this cohort, $82 \%$ of the patients had complete resolution of their symptoms. ${ }^{57}$ Martinez et al $^{58}$ reported their results on 131 endoscopic transaxillary FRR $(n=105)$ using two different robotic platforms. This technique required multiple incisions and collapse of the lung. The authors stated enhanced visualization allowing for more precise tissue manipulation and noted comparable results to conventional transaxillary FRR. ${ }^{58}$ Although further research with larger series is required to fully examine the safety and efficacy of minimally invasive techniques in the management of TOS, the existing published studies show promising results when compared to conventional approaches.

\section{Conclusions}

NTOS represents the most common subtype of TOS and can affect quality of life significantly. Minimal pathophysiologic advances have occurred which have hampered the diagnosis and treatment. Therefore, the diagnosis remains controversial, particularly due to its lack of objective findings, and nonspecific presentation. Many patients report significant delays in or an absence of a diagnosis, which naturally leads to frequent progression of their symptoms. The 2016 reporting standards suggest that the diagnosis of NTOS remains highly dependent on eliciting symptoms from a patient's history, and the physician's index of suspicion based on experience treating the syndrome. A significant update to the criteria used for establishing a diagnosis of NTOS includes a positive response to an injection of local anesthetic. ${ }^{59}$
Treatment includes BTX-A to the anterior scalene muscle, or the middle scalene or pectoralis minor muscles as well as surgery. The best available evidence for BTX-A therapy to the cervicothoracic muscles supports the value of this treatment for reducing pain in the affected extremity, and for an approximate duration of 2 months or more. Surgical approaches and newer minimally invasive surgical approaches offer high rates of improvement in select centers. An evidence-based approach to the sequence of treatments remains elusive, but many practitioners rely on physical therapy initially followed by BTXA interventions, and then decompressive surgery. Some physicians may offer surgery prior to BTX-A injections. Although decompressive surgery is considered the definitive therapy, there may be an increasing role for minimally invasive modalities as the etiology is better understood, and management evolves.

\section{Disclosure}

No potential conflicts of interest relevant to this article were reported.

\section{References}

1. Peet RM, Henriksen JD, Anderson TP, Martin GM. Thoracic-outlet syndrome: evaluation of a therapeutic exercise program. Proc Staff Meet Mayo Clin. 1956;31(9):281-287.

2. Ferrante MA, Ferrante ND. The thoracic outlet syndromes: part 1. overview of the thoracic outlet syndromes and review of true neurogenic thoracic outlet syndrome. Muscle Nerve. 2017;55(6):782-793. doi:10.1002/mus.25536

3. Klaassen Z, Sorenson E, Tubbs RS, et al. Thoracic outlet syndrome: a neurological and vascular disorder. Clin Anat. 2014;27(5):724. doi:10.1002/ca.22271

4. Illig KA, Donahue D, Duncan A, et al. Reporting standards of the society for vascular surgery for thoracic outlet syndrome. $J$ Vasc Surg. 2016;64(3):23.

5. Chang DC, Lisa A, Mukherjee D, De Leon R, Freischlag JA. Surgical intervention for thoracic outlet syndrome improves patient's quality of life. $J$ Vasc Surg. 2009;49(3):630-637. doi:10.1016/j. jvs.2008.10.023

6. Christo P, McGreevy K. Updated perspectives on neurogenic thoracic outlet syndrome. Curr Pain Headache Rep. 2011;15(1):14-21. doi:10.1007/s11916-010-0163-1

7. Illig KA, Rodriguez-Zoppi E. How common is thoracic outlet syndrome? Thorac Surg Clin. 2021;31(1):11-17. doi:10.1016/j. thorsurg.2020.09.001

8. Citisli V. Assessment of diagnosis and treatment of thoracic outlet syndrome, an important reason of pain in upper extremity, based on literature. J Pain Relief. 2015;4(2):1-7. doi:10.4172/2167-0846.1000173

9. Brantigan CO, Roos DB. Etiology of neurogenic thoracic outlet syndrome. Hand Clin. 2004;20(1):17. doi:10.1016/s0749-0712(03) 00112-4

10. Freischlag J, Orion K. Understanding thoracic outlet syndrome. Scientifica (Cairo). 2014;2014:248163-248166. doi:10.1155/2014/ 248163 
11. Sanders RJ, Hammond SL, Rao NM. Diagnosis of thoracic outlet syndrome. $J$ Vasc Surg. 2007;46(3):601-604. doi:10.1016/j. jvs.2007.04.050

12. Jones MR, Prabhakar A, Viswanath O, et al. Thoracic outlet syndrome: a comprehensive review of pathophysiology, diagnosis, and treatment. Pain Ther. 2019;8(1):5-18. doi:10.1007/s40122-019-0124-2

13. Atasoy E. Thoracic outlet syndrome: anatomy. Hand Clin. 2004;20 (1):7. doi:10.1016/s0749-0712(03)00078-7

14. Hooper TL, Denton J, McGalliard MK, Brismée J, Sizer PS. Thoracic outlet syndrome: a controversial clinical condition. part 1: anatomy, and clinical examination/diagnosis. J Man Manip Ther. 2010;18 (2):74-83. doi:10.1179/106698110X12640740712734

15. Huang JH, Zager EL. Thoracic outlet syndrome. Neurosurgery. 2004;55(4):897-903. doi:10.1227/01.NEU.0000137333.04342.4D

16. Hooper TL, Denton J, McGalliard MK, Brismée J, Sizer Jr PS Jr. Thoracic outlet syndrome: a controversial clinical condition. part 2: non-surgical and surgical management. J Man Manip Ther. 2010;18 (3):132-138. doi:10.1179/106698110X12640740712338

17. Kuhn J, Lebus G, Bible J. Thoracic outlet syndrome. J Am Acad Orthop Surg. 2015;23(4):222-232. doi:10.5435/JAAOS-D-13-00215

18. Machleder HI, Moll F, Verity MA. The anterior scalene muscle in thoracic outlet compression syndrome: histochemical and morphometric studies. Arch Surg. 1986;121(10):1141-1144. doi:10.1001/ archsurg.1986.01400100047009

19. Arányi Z, Csillik A, Böhm J, Schelle T. Ultrasonographic identification of fibromuscular bands associated with neurogenic thoracic outlet syndrome: the "wedge-sickle" sign. Ultrasound Med Biol. 2016;42(10):2357-2366. doi:10.1016/j.ultrasmedbio.2016.06.005

20. Doneddu PE, Coraci D, De Franco P, Paolasso I, Caliandro P, Padua L. Thoracic outlet syndrome: wide literature for few cases. status of the art. Neurol Sci. 2016;38(3):383. doi:10.1007/s10072016-2794-4

21. Baumer P, Baumer P, Kele H, et al. Thoracic outlet syndrome in $3 \mathrm{~T}$ MR neurography - fibrous bands causing discernible lesions of the lower brachial plexus. Eur Radiol. 2014;24(3):756-761. doi:10.1007/ s00330-013-3060-2

22. Swank RL, Simeone FA. The scalenus anticus syndrome: types; their characterization, diagnosis and treatment. Arch NeurPsych. 1944;51 (5):432-445. doi:10.1001/archneurpsyc.1944.02290290019004

23. Grunebach H, Arnold MW, Lum YW. Thoracic outlet syndrome. Vasc Med. 2015;20(5):493-495. doi:10.1177/1358863X15598391

24. Weaver ML, Hicks CW, Fritz J, Black JH, Lum YW. Local anesthetic block of the anterior scalene muscle increases muscle height in patients with neurogenic thoracic outlet syndrome. Ann Vasc Surg. 2019;59:28-35. doi:10.1016/j.avsg.2019.01.023

25. Panda N, Donahue DM. Evaluation of patients with neurogenic thoracic outlet syndrome. Thorac Surg Clin. 2021;31(1):55-59. doi:10.1016/j.thorsurg.2020.09.005

26. Povlsen S, Povlsen B. Diagnosing thoracic outlet syndrome: current approaches and future directions. Diagnostics (Basel). 2018;8(1):21. doi:10.3390/diagnostics 8010021

27. Kleinrensink GJ, Stoeckart R, Mulder PGH, et al. Upper limb tension tests as tools in the diagnosis of nerve and plexus lesions: anatomical and biomechanical aspects. Clin Biomech. 2000;15(1):9-14. doi:10.1016/S0268-0033(99)00042-X

28. Rayan G, Jensen C. Thoracic outlet syndrome: provocative examination maneuvers in a typical population. J Shoulder Elbow Surg. 1995;4(2):113-117. doi:10.1016/S1058-2746(05)80064-3

29. Gillard J, Pérez-Cousin M, Hachulla É, et al. Diagnosing thoracic outlet syndrome: contribution of provocative tests, ultrasonography, electrophysiology, and helical computed tomography in 48 patients. Joint Bone Spine. 2001;68(5):416-424. doi:10.1016/S1297-319X(01) 00298-6

30. Weaver ML, Lum YW. New diagnostic and treatment modalities for neurogenic thoracic outlet syndrome. Diagnostics (Basel). 2017;7 (2):28. doi:10.3390/diagnostics7020028
31. Singh VK, Jeyaseelan L, Kyriacou S, Ghosh S, Sinisi M, Fox M. Diagnostic value of magnetic resonance imaging in thoracic outlet syndrome. $J$ Orthop Surg. 2014;22(2):228-231. doi:10.1177/ 230949901402200224

32. Tsao BE, Ferrante MA, Wilbourn AJ, Shields RW. Electrodiagnostic features of true neurogenic thoracic outlet syndrome. Muscle Nerve. 2014;49(5):724-727. doi:10.1002/mus.24066

33. Gage M, Parnell H. SCALENUS ANTICUS SYNDROME* medicine and the section on general surgery, Ochsner clinic, new orleans. Am J Surg. 1947;73(2):252-268. doi:10.1016/0002-9610(47)903206

34. Jordan SE, Ahn SS, Gelabert HA. Combining ultrasonography and electromyography for botulinum chemodenervation treatment of thoracic outlet syndrome: comparison with fluoroscopy and electromyography guidance. Pain Physician. 2007;10(4):541-546. doi:10.36076/ppj.2007/10/541

35. Torriani M, Gupta R, Donahue D. Sonographically guided anesthetic injection of anterior scalene muscle for investigation of neurogenic thoracic outlet syndrome. Skeletal Radiol. 2009;38(11):1083-1087. doi:10.1007/s00256-009-0714-x

36. Christo PJ, Christo DK, Carinci AJ, Freischlag JA. Single CT-guided chemodenervation of the anterior scalene muscle with botulinum toxin for neurogenic thoracic outlet syndrome. Pain Med. 2010;11 (4):504-511. doi:10.1111/j.1526-4637.2010.00814.x

37. Braun RM, Sahadevan DC, Feinstein J. Confirmatory needle placement technique for scalene muscle block in the diagnosis of thoracic outlet syndrome. Tech Hand Up Extrem Surg. 2006;10(3):173-176. doi:10.1097/01.bth.0000231967.74041.48

38. Jordan SE, Machleder HI. Diagnosis of thoracic outlet syndrome using electrophysiologically guided anterior scalene blocks. Ann Vasc Surg. 1998;12(3):260-264. doi:10.1007/s100169900150

39. Mashayekh A, Christo PJ, Yousem DM, Pillai JJ. CT-guided injection of the anterior and middle scalene muscles: technique and complications. AJNR Am J Neuroradiol. 2011;32(3):495-500. doi:10.3174/ajnr.A2319

40. Donahue DM, Godoy IRB, Gupta R, Donahue JA, Torriani M. Sonographically guided botulinum toxin injections in patients with neurogenic thoracic outlet syndrome: correlation with surgical outcomes. Skeletal Radiol. 2020;49(5):715-722. doi:10.1007/ s00256-019-03331-9

41. Collins E, Orpin M. Physical therapy management of neurogenic thoracic outlet syndrome. Thorac Surg Clin. 2021;31(1):61-69. doi:10.1016/j.thorsurg.2020.09.003

42. Balderman J, Abuirqeba AA, Eichaker L, et al. Physical therapy management, surgical treatment, and patient-reported outcomes measures in a prospective observational cohort of patients with neurogenic thoracic outlet syndrome. J Vasc Surg. 2019;70(3):832-841. doi:10.1016/j.jvs.2018.12.027

43. Godoy IRB, Donahue DM, Torriani M. Botulinum toxin injections in musculoskeletal disorders. Semin Musculoskelet Radiol. 2016;20 (5):441. doi:10.1055/s-0036-1594284

44. Torriani M, Gupta R, Donahue DM. Botulinum toxin injection in neurogenic thoracic outlet syndrome: results and experience using a ultrasound-guided approach. Skeletal Radiol. 2010;39(10):973. doi:10.1007/s00256-010-0897-1

45. Aoki KR. Review of a proposed mechanism for the antinociceptive action of botulinum toxin type A. Neurotoxicology. 2005;26 (5):785-793.

46. Sheean G. Botulinum toxin for the treatment of musculoskeletal pain and spasm. Curr Pain Headache Rep. 2002;6(6):460-469. doi:10.1007/s11916-002-0065-y

47. Finlayson HC, O'Connor RJ, Brasher PMA, Travlos A. Botulinum toxin injection for management of thoracic outlet syndrome: a double-blind, randomized, controlled trial. Pain (Amsterdam). 2011;152(9):2023-2028. doi:10.1016/j.pain.2011.04.027 
48. Freischlag JA. A decade of excellent outcomes after surgical intervention: 538 patients with thoracic outlet syndrome. Trans Am Clin Climatol Assoc. 2018;129:88-94.

49. Roos DB. Transaxillary approach for first rib resection to relieve thoracic outlet syndrome. Ann Surg. 1966;163(3):354-358. doi:10.1097/00000658-196603000-00005

50. Likes KC, Orlando MS, Salditch Q, et al. Lessons learned in the surgical treatment of neurogenic thoracic outlet syndrome over 10 years. Vasc Endovascular Surg. 2015;49(1-2):8-11. doi:10.1177/ 1538574415583850

51. Moore R, Wei Lum Y. Venous thoracic outlet syndrome. Vasc Med. 2015;20(2):182-189. doi:10.1177/1358863X14568704

52. Doyle A, Wolford HY, Davies MG, et al. Management of effort thrombosis of the subclavian vein: today's treatment. Ann Vasc Surg. 2007;21(6):723-729.

53. Lee JT, Karwowski JK, Harris EJ, Haukoos JS, Olcott C. Long-term thrombotic recurrence after nonoperative management of Paget-Schroetter syndrome. J Vasc Surg. 2006;43(6):1236-1243.

54. Archie MM, Gelabert HA. Endovascular reconstruction of subclavian artery aneurysms in patients with arterial thoracic outlet syndrome. Ann Vasc Surg. 2019;57:10-15.
55. Soukiasian HJ, Shouhed D, Serna-Gallgos D, McKenna R, Bairamian VJ. A video-assisted thoracoscopic approach to transaxillary first rib resection. Innovations (Phila). 2015;10(1):21-26. doi:10.1097/IMI.0000000000000120

56. Gharagozloo F, Meyer M, Tempesta B, Gruessner S. Robotic transthoracic first-rib resection for Paget-Schroetter syndrome. Eur $J$ Cardiothorac Surg. 2019;55(3):434-439. doi:10.1093/ejcts/ezy275

57. Abdellaoui A, Atwan M, Reid F, Wilson P. Endoscopic assisted transaxillary first rib resection. Interact Cardiovasc Thorac Surg. 2007;6(5):644-646. doi:10.1510/icvts.2007.151423

58. Martinez BD, Wiegand CS, Evans P, Gerhardinger A, Mendez J. Computer-assisted instrumentation during endoscopic transaxillary first rib resection for thoracic outlet syndrome: a safe alternate approach. Vascular. 2005;13(6):327-335. doi:10.1258/rsmvasc.1 3.6.327

59. Illig KA. Neurogenic thoracic outlet syndrome: bringing order to chaos. J Vasc Surg. 2018;68(4):939-940. doi:10.1016/j.jvs.201 8.04 .030

\section{Publish your work in this journal}

The Journal of Pain Research is an international, peer reviewed, open access, online journal that welcomes laboratory and clinical findings in the fields of pain research and the prevention and management of pain. Original research, reviews, symposium reports, hypothesis formation and commentaries are all considered for publication. The manuscript management system is completely online and includes a very quick and fair peer-review system, which is all easy to use. Visit http:// www.dovepress.com/testimonials.php to read real quotes from published authors. 